\title{
Novel Traveling Hyperbolic Function Solution for Evolution Equation
}

\author{
Hong-Lei Wang ${ }^{1,}$, , Chun-Huan Xiang ${ }^{2, b}$
}

${ }^{1}$ College of medical informatics, Chongqing Medical University, Chongqing, 400016, P. R. China

${ }^{2}$ School of Public Health and Management, Chongqing Medical University, Chongqing, 400016, P.R. China

aemail:w825900@163.com, bemail:w8259300@126.com

Keywords: nonlinear, Ostrovsky equation, traveling wave solution, evolution equation

Abstract. The discrete nonlinear evolution equation concerned is the nonlinear Ostrovsky equation, which is employed in engineering and physics to describe some nonlinear evolution phenomena such as the temperature transmission and investigated by expand function method. The novel traveling wave solutions are obtained. The results are simply discussed.

\section{Introduction}

When a stationary solution loses its stability as a physical parameter crosses a critical value, a periodic solution can arise. The existence of periodic solutions in nonlinear differential equations is an interesting topic in many applications, such as problems in biological and control systems. Nonlinear equations are very important in describe evolution phenomena in engineering and physics. People have investigated many useful methods to obtain the evolution solutions: the inverse scattering method [1-3], the Hirota's bilinear method [4-6], the Darboux and Backlund transformation [7,8]. Sine-cosine method $[9,10]$. The expand method is one of the powerful methods to obtain the traveling hyperbolic function solution. The nonlinear Ostrovsky equation, which is an important nonlinear equation in physics andengineer, is investigated and the numerical solutions are obtained.

\section{The Nonlinear Ostrovsky Equation and Expand Method}

The nonlinear evolution equation, which was first introduced by Ostrovsky[11,12] to describe nonlinear surface and internal waves in rotating ocean, is in the form as follow

$$
\left(u_{t}+c_{0} u_{x}+p u u_{x}+q u_{x x x}\right)_{x}=\gamma
$$

Where ${ }^{c_{0}}$ is the velocity of dispersionless linear waves, $\mathrm{p}$ is the nonlinear coefficient, $\mathrm{q}$ is the Boussinesq dispersion and $\gamma$ is the Coriolis dispersion coefficients. Coriolis dispersion is negligible $(\gamma=0)$, Eq. (1) reduces to the $\mathrm{KdV}$ equation. As a limiting case of very long waves for which Boussinesq dispersion vanishes $\left(\mathrm{q}^{=0}\right)$. The reduced equation is rewritten as[13-15]

$$
u u_{x x t}-u_{x} u_{x t}+u^{2} u_{t}=0
$$

For a Nonlinear equation evolution with time $\mathrm{t}$ and dimension $x$, the general form is given as

$$
G\left(u, u_{x}, u_{t}, u_{x x}, u_{t t}, u_{x t}, \ldots\right)=0
$$

In order obtain the solutions of $\mathrm{Eq}(3)$, we employ an expression and simplify the equation as

$$
G\left(u, u_{\xi}, u_{\xi \xi}, u_{\xi \xi \xi}, \ldots\right)=0
$$

with the expression as $\xi=\alpha(x-\beta t)$. The minimal expand is used to obtain the approximation evolution solution for nonlinear equation.

Numerical Example. We employ the minimal expand method for the nonlinear equations (2), the useful expression is used. Submitting the equation $\xi=\alpha(x-\beta t)$ into Eq (2), We obtain

$$
\alpha^{2} u u_{\xi \xi \xi}-\alpha^{2} u_{\xi} u_{\xi \xi}+u^{2} u_{\xi}=0
$$


Integrating once $\mathrm{Eq}(4)$, the equation is changed into

$$
3 \alpha^{2} u u_{\xi \xi}-3 \alpha^{2} u_{\xi} u_{\xi}+u^{3}=0
$$

Generally, the hyperbolic function solution of Eq.(5) is written as

$$
u=g+f \sec h \xi
$$

where

$$
\begin{aligned}
& g=A_{0}+A_{1} \tanh \xi+A_{2} \tanh \xi^{2} \\
& f=B_{0}+B_{1} \tanh \xi
\end{aligned}
$$

with $A_{0}, A_{1}, A_{2}, B_{0}$ and $B_{1}$ are parameters to be determined.

The derivative for Eq.(7) $g$ and Eq.(8) $f$ are given as the follow

$$
\begin{aligned}
& g_{\xi}=A_{1}\left(1+\tanh \xi^{2}\right)+2 A_{2} \tanh \xi\left(1+\tanh \xi^{2}\right) \\
& f_{\xi}=B_{1}\left(1+\tanh \xi^{2}\right) \\
& g_{\xi \xi}=-2 A_{1}\left(1+\tanh \xi^{2}\right) \tanh \xi+A_{2}\left(2\left(1+\tanh \xi^{2}\right)^{2}-4\left(1+\tanh \xi^{2}\right) \tanh \xi^{2}\right) \\
& f_{\xi \xi}=-2 B_{1}\left(1+\tanh \xi^{2}\right) \tanh \xi
\end{aligned}
$$

Submitting the equation $g, f, g_{\xi}, f_{\xi}, g_{\xi \xi}, f_{\xi \xi}$ into $u$, we obtain the expression $u, u_{\xi}, u_{\xi \xi}$. Then, we put the function $u, u_{\xi}, u_{\xi \xi}$ into Eq.(5) and equating the coefficient of $\operatorname{Sech} \xi$ to zero, we obtain the following equation(the relation $1+\tanh \xi^{2}=\sec h \xi^{2}$ is employed ):

$$
\begin{aligned}
& A_{0}^{3}-3 A_{0} A_{1}^{2}-3 A_{0}^{2} A_{2}+3 A_{1}^{2} A_{2}+3 A_{0} A_{2}^{2}-A_{2}^{3}=0 \\
& -3 a^{2} A_{0} B_{0}+3 A_{0}^{2} B_{0}-3 B_{0} A_{1}^{2}+3 a^{2} A_{2} B_{0}-6 A_{0} A_{2} B_{0}+3 A_{2}^{2} B_{0}+3 a^{2} B_{1} A_{1}-6 A_{0} A_{1} B_{1}+6 A_{1} A_{2} B_{1}=0 \\
& 6 a^{2} A_{1}^{2}+3 A_{1}^{2} A_{0}+12 a^{2} A_{0} A_{2}+3 A_{0}^{2} A_{2}-6 A_{1}^{2} A_{2}-12 a^{2} A_{2}^{2}-6 A_{2}^{2} A_{0}+3 A_{2}^{3}+3 A_{0} B_{0}^{2}-3 A_{2} B_{0}^{2} \\
& -6 A_{1} B_{0} B_{1}-3 A_{0} B_{1}^{2}+3 A_{2} B_{1}^{2}=0 \\
& 3 A_{1}^{2} B_{0}-3 a^{2} A_{2} B_{0}+6 A_{0} A_{2} B_{0}-6 A_{2}^{2} B_{0}+B_{0}^{3}+9 a^{2} A_{1} B_{1}+6 A_{0} A_{1} B_{1}-12 A_{1} A_{2} B_{1}-3 B_{1}^{2} B_{0}=0 \\
& -9 a^{2} A_{1}^{2}-6 a^{2} A_{2} A_{0}+3 A_{1}^{2} A_{2}+30 A_{2}^{2} a^{2}+3 A_{2}^{2} A_{0}-3 A_{2}^{3}-3 a^{2} B_{0}^{2}+3 A_{2} B_{0}^{2}+6 A_{1} B_{0} B_{1}+9 a^{2} B_{1}^{2} \\
& +3 A_{0} B_{1}^{2}-6 A_{2} B_{1}^{2}=0 \\
& 6 a^{2} A_{2} B_{0}+3 A_{2}^{2} B_{0}-18 a^{2} A_{1} B_{1}+6 A_{1} A_{2} B_{1}+3 B_{1}^{2} B_{0}=0 \\
& -18 a^{2} A_{2}^{2}+A_{2}^{3}-12 a^{2} B_{1}^{2}+3 B_{1}^{2} A_{2}=0
\end{aligned}
$$

To obtain the solution for the above equation, $A_{0}=0$ is supposed to be given. Then, we obtain the follow results:

$$
\begin{gathered}
A_{2}=\sqrt{3} A_{1} \\
B_{0}=-\frac{a^{2} \sqrt{3}+2 \sqrt{3} A_{1}}{a^{2} \sqrt{3}+2 A_{1}} B_{1} \\
B_{1}= \pm \sqrt{\frac{-6 a^{4} A_{1}(1+\sqrt{3})-12 a^{2} A_{1}^{2}-3 \sqrt{3} a^{2} A_{1}^{2}-2 \sqrt{3} A_{1}^{3}-6 a^{2} A_{1}^{2}}{\sqrt{3}\left(a^{2}+2 A_{1}\right)}}
\end{gathered}
$$

Submitting $A_{0}, A_{1}, A_{2}, B_{0}$ and $B_{1}$ into Eq.(6), we obtain the evolution solutions as the follow 


$$
\begin{aligned}
& u=A_{1} \tanh \xi+\sqrt{3} A_{1} \tanh \xi^{2}+\left[\left(-\frac{a^{2} \sqrt{3}+2 \sqrt{3} A_{1}}{a^{2} \sqrt{3}+2 A_{1}}\right)\right. \\
& \left(\sqrt{\frac{-6 a^{4} A_{1}(1+\sqrt{3})-12 a^{2} A_{1}^{2}-3 \sqrt{3} a^{2} A_{1}^{2}-2 \sqrt{3} A_{1}^{3}-6 a^{2} A_{1}^{2}}{\sqrt{3}\left(a^{2}+2 A_{1}\right)}}\right) \\
& \left.+\sqrt{\frac{-6 a^{4} A_{1}(1+\sqrt{3})-12 a^{2} A_{1}^{2}-3 \sqrt{3} a^{2} A_{1}^{2}-2 \sqrt{3} A_{1}^{3}-6 a^{2} A_{1}^{2}}{\sqrt{3}\left(a^{2}+2 A_{1}\right)}} \tanh \xi\right] \sec h \xi
\end{aligned}
$$

The evolution solution figure for Eq.(9) and (10) are given in Fig.1 and Fig.2, respectively.

$$
\begin{aligned}
& u(x, t)=A_{1} \tanh \alpha(x-\beta t)+\sqrt{3} A_{1} \tanh (\alpha(x-\beta t))^{2}+\left[\left(\frac{a^{2} \sqrt{3}+2 \sqrt{3} A_{1}}{a^{2} \sqrt{3}+2 A_{1}}\right)\right. \\
& \left(\sqrt{\frac{-6 a^{4} A_{1}(1+\sqrt{3})-12 a^{2} A_{1}^{2}-3 \sqrt{3} a^{2} A_{1}^{2}-2 \sqrt{3} A_{1}^{3}-6 a^{2} A_{1}^{2}}{\sqrt{3}\left(a^{2}+2 A_{1}\right)}}\right) \\
& \left.-\sqrt{\frac{-6 a^{4} A_{1}(1+\sqrt{3})-12 a^{2} A_{1}^{2}-3 \sqrt{3} a^{2} A_{1}^{2}-2 \sqrt{3} A_{1}^{3}-6 a^{2} A_{1}^{2}}{\sqrt{3}\left(a^{2}+2 A_{1}\right)}} \tanh \alpha(x-\beta t)\right] \operatorname{sech} \alpha(x-\beta t) \\
& x \in(-12,12), t \in(-12,12)
\end{aligned}
$$

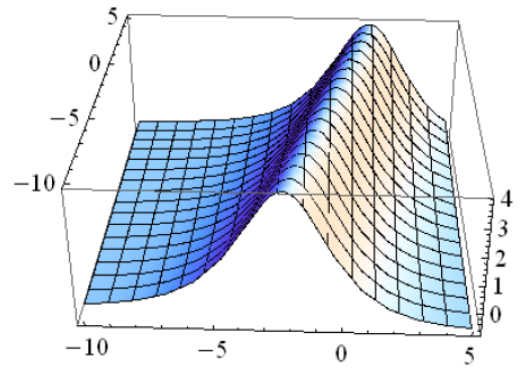

\section{Conclusions}

Nonlinear equations are very important in describe evolution phenomena in engineering and physics. The discrete nonlinear evolution equation concerned is the nonlinear Ostrovsky equation, which is employed in engineering and physics to describe some nonlinear evolution phenomena such as the temperature transmission and investigated by expand function method. The novel traveling wave solutions are obtained. The simulation figures for the evolution solutions are shown.

\section{References}

[1] G. Neugebauer, Progress in relativistic gravitational theory using the inverse scattering method, Journal of Mathematical Physics, 44(8):3407-3429, 2003.

[2] V.O. Vakhnenko, E.J. Parkes, The singular solutions of a nonlinear evolution equation taking continuous part of the spectral data into account in inverse scattering method, Chaos Solitons \& Fractals, 45(6):846-852, 2012. 
[3] M.J. Ablowitz, P.A. Clarkson, Solitons, Nonlinear Evolution Equations and Inverse Scattering Transform, Cambridge University Press, Cambridge, 1990.

[4] G.F. Yu, D. Lao, Complex and coupled complex negative order AKNS equation, Communications in Nonlinear Science \& Numerica,30(1-3):196-206, 2016.

[5] X.H. Meng, Wronskian and Grammian determinant structure solutions for a variable-coefficient forced Kadomtsev-Petviashvili equation in fluid dynamics, Physica A Statistical Mechanics \& Its Application, 413:635-642, 2014.

[6] R. Hirota, Direct method of finding exact solutions of nonlinear evolution equations, in: R. Bullough, P. Caudrey (Eds.), Blacklund Transformations, Springer, Berlin, 1980, pp. 1157-1175.

[7] L.L. Xue, Q.P. Liu, Bäcklund-Darboux Transformations and Discretizations of Super KdV Equation, Symmetry Integrability \& Geometry Methods \& Application, 10:045, 2013.

[8] I.M. Anderson, M.E. Fels, Backlund Transformations for Darboux Integrable Differential Systems: Examples and Applications, Journal of Geometry \& Physics, 102:1-31, 2014.

[9] A.M. Wazwaz, The tanh and the sine-cosine methods for a reliable treatment of the modified equal width equation and its variants, Commun. Nonlinear Sci. Numer Simul. 11 (2),148-160, (2006).

[10] A.M. Wazwaz, The tanh method and the sine-cosine method for solving the KP-MEW equation, Int. J. Comput. Math. 82 (2), 235-246, (2005).

[11] E. Yusufoflu, A. Bekir, A traveling wave solution to the Ostrovsky equation, Appl. Math. Comput. 186, 256-260, (2007).

[12] L.A. Ostrovsky, Nonlinear internal waves in a rotating ocean, Oceanology 18, 119-125, (1978).

[13] V.O. Vakhnenko, E.J. Parkes, The two loop soliton of the Vakhnenko equation, Nonlinearity 11, 1457-1464, (1998).

[14] V.O. Vakhnenko, E.J. Parkes, The calculation of multi-soliton solutions of Vakhnenko equation, Chaos Solitons Fractals, 13, 1819-1826, (2002)

[15] C. Köroflu, T. Özi, A novel traveling wave solution for Ostrovsky equation using Exp-function method, Computers and Mathematics with Applications 58, 2142-2146, (2009). 